\title{
Pêche Et Caractéristiques Morphologiques D'un Poisson-Chat Endémique Synodontis Punctifer (Daget, 1964) Dans Le Barrage Électrique De Taabo (Côte d'Ivoire)
}

\section{Hervé Oussou Koffi}

Université Nangui Abrogoua, Unité de Formation et de Recherche des Sciences de la Nature (UFR-SN), Laboratoire de Biologie et de Cytologie Animale, Abidjan, Côte d'Ivoire

\section{Diaha N'guessan Constance}

Centre de Recherche Océanologique, Abidjan, Côte d'Ivoire

\section{Soro Yaya \\ N'Da Konan}

Université Nangui Abrogoua, Unité de Formation et de Recherche des Sciences de la Nature (UFR-SN), Laboratoire de Biologie et de Cytologie Animale, Abidjan, Côte d'Ivoire

Doi: 10.19044/esj.2018.v14n15p101 URL:http://dx.doi.org/10.19044/esj.2018.v14n15p101

\begin{abstract}
A study of Synodontis punctifer (family: Mochokidae) of Taabo Lake was conducted from October 2015 to September 2016. This was carried out so as to evaluate its quantity (biomass) caught by fishing and also its morphological characteristics. The fish were caught using gillnets at 4 sites of the lake and different measurements were made. The fishing effort was 23 to 11 fishermen / day in Taabo city, 17 to 11 fishermen / day in Courandjourou, 13 to 5 fishermen / day in Ahondo, and 8 to 3 fishermen / day in Taabo village. The catch per unit effort (CPUE) ranged from 17.7 to $3.7 \mathrm{~g} /$ fisherman / day, 9.2 to $1.2 \mathrm{~g} /$ fisherman / day, 8.3 to $0.7 \mathrm{~g} /$ fisherman /day, 8 to $1 \mathrm{~g} /$ fisherman / day at Ahondo, Courandjourou, Taabo city and Taabo village, respectively. The overall annual production was estimated at $2199.3 \mathrm{~kg}$, i.e. $817 \mathrm{~kg}(40 \%)$ at Ahondo, $788.3 \mathrm{~kg} \mathrm{(35 \% )} \mathrm{at} \mathrm{Courandjourou,} 462.5 \mathrm{~kg} \mathrm{(20 \% )}$ at Taabo, and $131.5 \mathrm{~kg}(5 \%)$ in Taabo village. The overall sex ratio (1: 1.33) was in favor of females. Individuals have negative allometric growth at all sites with values ranging from 1.72 to 2.89 . The condition factor does not vary significantly between different categories of individuals (ANOVA, $F=2.79, p>0.05$ ). A significant difference was observed between the average sizes of the individuals of the different sites (test, $\mathrm{p}<0,05$ ). The large size specimens were
\end{abstract}


captured at Ahondo, mean (avg $=15.38 \pm 1.68 \mathrm{~cm} \mathrm{FL).} \mathrm{In} \mathrm{Courandjourou,} \mathrm{the}$ sizes were intermediate $(\mathrm{avg}=13.63 \pm 1.64 \mathrm{~cm} \mathrm{LF})$. Smalls sizes have been observed in Taabo city $($ mean $=12.37 \pm 1.24 \mathrm{~cm} \mathrm{FL})$ and Taabo village (mean= $15.58 \pm 1.58 \mathrm{~cm}$ FL).

Keywords: Fishing, allometry, sex ratio, size structure, condition coefficient, Synodontis punctifer, Taabo lake, Ivory Coast

\section{Résumé}

Une étude sur Synodontis punctifer (famille : Mochokidae) du lac de Taabo a été réalisée d'octobre 2015 à septembre 2016, en vue d'évaluer sa quantité (biomasse) capturée par la pêche et ses caractéristiques morphologiques. Les poissons ont été pêchés par des filets maillants dans 4 sites du lac et les différentes mensurations effectuées. L'effort de pêche a été de 23 à 11 pêcheurs/ j à Taabo cité, 17 à 11 pêcheurs/ j à Courandjourou, 13 à 5 pêcheurs/ $\mathrm{j}$ à Ahondo et 8 à 3 pêcheurs/ $\mathrm{j}$ à Taabo village. La prise par unité d'effort (PUE) a varié de 17,7 à 3,7 g/ pêcheur/ j, 9,2 à 1,2 g/ pêcheur/ j, 8,3 à $0,7 \mathrm{~g} /$ pêcheur/ j, 8 à 1g/ pêcheur/ j respectivement à Ahondo, Courandjourou, Taabo cité et Taabo village. La production annuelle globale a été estimée à $2199,3 \mathrm{~kg}$ soit $817 \mathrm{~kg}(40 \%)$ à Ahondo, 788,3 kg (35\%) à Courandjourou, $462,5 \mathrm{~kg}(20 \%)$ à Taabo cité et $131,5 \mathrm{~kg}(5 \%)$ à Taabo village. Le sexe ratio globale $(1: 1,33)$ a été en faveur des femelles. Les individus ont une croissance allométrique négative sur l'ensemble des sites avec des valeurs oscillant entre 1,72 et 2,89 . Le facteur de condition ne varie pas de manière significative entre les différentes catégories d'individus (ANOVA, $\mathrm{F}=2,79 ; \mathrm{p}>0,05$ ). Une différence significative a été observée entre les tailles moyennes des individus des différents sites (test, $\mathrm{p}<0,05$ ). Les spécimens des grandes tailles ont été capturés à Ahondo, moyenne (moy= 15,38 $\pm 1,68 \mathrm{~cm} \mathrm{LF}$ ). A Courandjourou les tailles ont été intermédiaires (moy=13,63 $\pm 1,64 \mathrm{~cm}$ LF). Par contre les individus ont été de petites tailles à Taabo cité $(\mathrm{moy}=12,58 \mathrm{~cm}$ LF) et à Taabo village $(\operatorname{moy}=12,35 \mathrm{~cm} \mathrm{LF})$.

Mots-clés : pêche, allométrie, sexe ratio, structure de taille, coefficient de condition, Synodontis punctifer, lac Taabo, Côte d'Ivoire

\section{Introduction}

La Côte d'Ivoire est située en Afrique de l'ouest $\left(04^{\circ}-11^{\circ}\right.$ latitude nord et $03^{\circ}-08^{\circ}$ longitude ouest (Diaha et al., 2009). Elle dispose de 4 principaux lacs (Taabo, Kossou Buyo et Ayamé) sur lesquels se pratique l'ensemble des activités de la pêche continentale (Da Costa \& Dietoa, 2007). Les produits de cette pêche notamment le poisson, représente la principale source de protéines (plus de 70\%) pour les populations africaines (Micha \& Frank, 2004) et 
souvent l'unique source de protéines pour les populations à faibles revenus économiques. Le poisson contribue aussi à la prospérité des populations riveraines. Face à ses atouts indéniables et à l'augmentation rapide de la population ivoirienne, la pression s'est accrue sur les différentes pêcheries, créant ainsi des situations de surexploitation (Lévêque \& Paugy, 1999). Une pêche intensive entraîne la diminution de l'abondance et des rendements des opérations de pêche, une réduction de la taille de maturité des individus débarqués et une modification des captures au profit d'espèces moins prisées (Gracia \& Demetropoulos, 1986). Or Synodontis punctifer, qui est un poisson endémique à la Côte d'Ivoire rencontré dans le lac Taabo mais aussi dans les fleuves Bandama et Sassandra, est massivement capturé par les pêcheurs artisans de façon anarchique car il n'existe aucune base de gestion. Les travaux réalisés restent parcellaires.

Cette présente étude a pour objectif d'évaluer le niveau de production de l'espèce par la pêche artisanale et déterminer les caractéristiques morphologiques de sa population dans le lac de Taabo.

\section{Matériels et méthodes \\ Milieu d'étude}

Le lac Taabo $\left(06^{\circ} 20^{\prime} \mathrm{N}-06^{\circ} 40^{\prime} \mathrm{N}\right.$ et $\left.5^{\circ} \mathrm{O}-5^{\circ} 30^{\prime} \mathrm{O}\right)$ créé en 1978 sur le fleuve Bandama est situé à environ $110 \mathrm{~km}$ du confluent du Bandama blanc et du Bandama rouge, à $120 \mathrm{~km}$ en aval du lac Kossou et à $195 \mathrm{~km}$ de son embouchure (Kouassi et al., 2007 ; Aliko et al., 2010). Il couvre une superficie de $69 \mathrm{~km}^{2}$ à la côte de retenue. Son bassin versant est de $58700 \mathrm{~km}^{2}$ avec un débit annuel de $128,7 \mathrm{~m}^{3} / \mathrm{s}$ (Figure 1). Le climat est de type attiéen avec un régime hydraulique équatorial de transition. Deux types de végétation sont rencontrés. L'une pré forestière au nord et l'autre forestière à savanes incluses au sud (Groga, 2012). On distingue deux saisons des pluies (une grande allant d'avril à juin, une petite de septembre à novembre) et deux saisons sèche (la plus longue de décembre à mars et la petite de juillet à septembre (Berté et al., 2008). En outre, une grande partie du lac est occupée par des végétaux aquatiques (Aliko et al., 2010). 


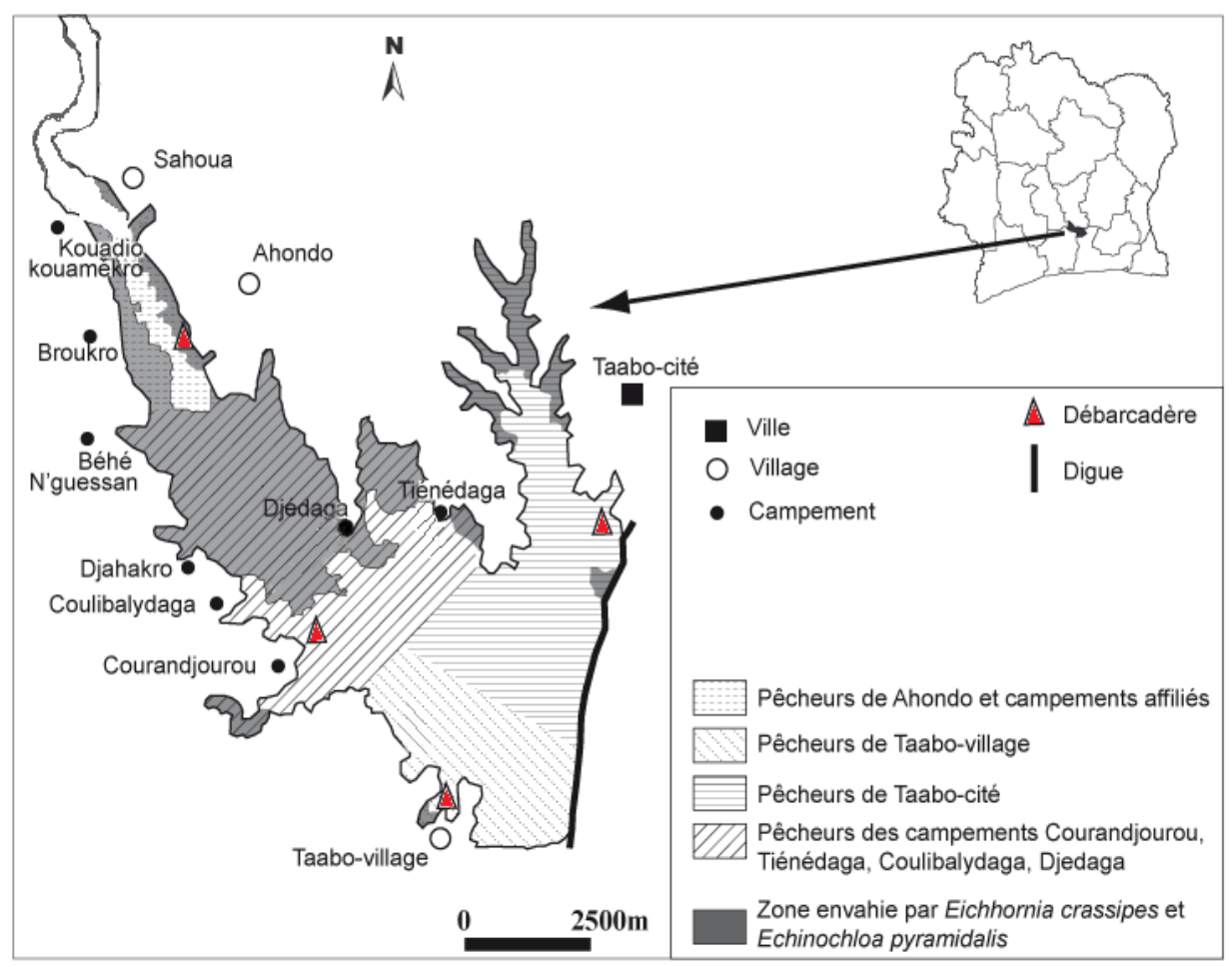

Figure 1. Barrage hydroélectrique de Taabo (Koudou, 2014)

\section{Pêche et échantillonnage}

D'octobre 2015 à septembre 2016, un échantillonnage de S. punctifer issu de la pêche artisanale a été réalisé chaque mois à raison d'une semaine par site. Au débarquement, un questionnaire a été donné à l'équipage en vue de collecter des informations sur le nom du pêcheur, l'engin utilisé, les dimensions de l'engin et la zone de pêche. Les poissons ont été triés et la masse totale évaluée avec une balance de $10 \mathrm{~kg}$ de portée et d'une précision de $1 \mathrm{~g}$.

Les spécimens ont été ensuite choisis au hasard dans les différents lots. Ainsi, 1160 individus ont été échantillonnés sur l'ensemble des sites en raison de 375 à Ahondo, 247 à Courandjourou, 276 à Taabo village et 262 à Taabo cité. Chaque individu échantillonné a été mesuré (longueur totale LT et longueur à la fourche LF, au centimètre près à l'aide d'un ichtyomètre), pesé (poids total PT, avec une balance de $500 \mathrm{~g}$ de portée et d'une précision de 0,01 $\mathrm{g}$ ), disséqué puis le sexe a été déterminé. L'activité de pêche commence en général dans la soirée avec la pose des filets et se termine le lendemain au retrait des filets. L'engin de pêche principalement utilisé a été le filet maillant de longueur variant entre 100 et $800 \mathrm{~m}$, de profondeur $150 \mathrm{~m}$ et de maillage de $5,10,15,20,25,30,35,40,45,50,55,60 \mathrm{~mm}$ entre nœud. 


\section{Méthode d'exploitation des données}

Estimation de l'effort et des captures par unité d'effort et des captures globales de pêche

L'effort de pêche est un indice de population et peut être défini comme étant le nombre de pêcheurs en activité par unité de surface (Laë, 1997). Cette variable est la mieux indiquée à la pêche artisanale tropicale vue l'aspect hétérogène des comportements de pêche.

Dans notre étude, l'unité d'effort de pêche a été estimée par le nombre de pêcheurs en activité de pêche au cours d'une journée donnée. La prise par unité d'effort (PUE) exprimée en g est le poids des captures réalisées par pêcheur durant une journée de pêche. Pour chaque mois, une PUE moyenne par site a été déterminée à partir de l'ensemble des captures journalières enregistrées durant le mois considéré selon la formule suivante :

PUEm = Pt/ Nc (Tah et $a l ., 2009)$

PUEm = prise moyenne mensuelle par unité d'effort en $\mathrm{g} ; \mathrm{Pt}=$ poids total frais en $\mathrm{kg}$ des captures débarquées par l'ensemble des pêcheurs enquêtés dans le mois par site $; \mathrm{Nc}=$ nombre total de pêcheurs enquêtés par site dans le mois.

Les captures totales mensuelles $(\mathrm{Ci})$ sont estimées par extrapolation selon la formule suivante :

Ci $=$ PUE mi $\times$ Fmi $\times \mathbf{j}($ Tah et $a l ., 2009)$

$\mathrm{Ci}=$ capture globale $(\mathrm{kg}) \mathrm{du}$ mois $\mathrm{i}$; PUE mi $=$ prise moyenne mensuelle par unité d'effort en $\mathrm{g}$ au cours du mois $\mathrm{i}$; Fmi = effort de pêche mensuel exprimé en nombre moyen de pêcheurs débarquant par jour dans le mois $\mathrm{i} ; \mathrm{j}=$ nombre de jours de pêche dans le mois $\mathrm{i}$.

Les captures annuelles $(\mathrm{Ca})$ dans les différents sites d'étude sont déterminées en faisant la somme des captures mensuelles selon la formule suivante :

$$
\mathbf{C a}=\mathbf{\Sigma C i}(\text { Da Costa \& Dietao, 2007) }
$$

La production globale de poissons du lac est déterminée à partir de l'équation suivante :

$\mathbf{P g}=\mathbf{P U E m a} \times \mathbf{F t} \times \mathbf{J}($ Gerlotto $\&$ Stequert, 1978)

$\mathrm{Pg}=$ production globale de poissons du lac pour un cycle annuel ; PUEma = prise moyenne annuelle par unité d'effort sur l'ensemble du lac calculée à partir des PUEm obtenues dans les quatre sites échantillonnés ; J = nombre de jours de pêche durant le cycle annuel d'étude.

\section{Détermination des caractéristiques des populations}

\section{- Sexe ratio}

Le sexe ratio $(\mathrm{SR})$ est le nombre de femelles $(\mathrm{F})$ pour un mâle $(\mathrm{M})$ dans une population donnée. Elle est déterminée par la formule suivante :

$\mathbf{S R}=\mathbf{F} / \mathbf{M}($ Kartas \& Quignard, 1984) 
- $\quad$ Facteur de condition

Le coefficient de condition (Kc) permet de déterminer l'embonpoint des poissons dans un milieu. La formule est :

$$
\mathbf{K c}=\left(\mathbf{P T} / \mathbf{L F}^{\mathbf{3}}\right) \times \mathbf{1 0}^{\mathbf{5}}(\text { Kraidy et } \text { al. }, 2014)
$$

$\mathrm{PT}=$ poids du poisson $(\mathrm{g}) ; \mathrm{LF}=$ longueur à la fourche $(\mathrm{mm})$

\section{- Relation allométrique}

Les relations taille-poids obéissent au modèle :

$$
\mathbf{P}=\mathbf{a L F}^{\mathbf{b}} \text { (Le Cren, 1951) }
$$

$\mathrm{P}=$ poids du spécimen $(\mathrm{g}) ; \mathrm{LF}=$ longueur à la fourche $(\mathrm{cm})$.

Les paramètres $\mathrm{a}$ et $\mathrm{b}$ représentent respectivement le coefficient de proportionnalité et le coefficient allométrique et sont déterminés après la transformation logarithmique de type:

$$
\operatorname{Ln}(\mathbf{P})=\ln (\mathbf{a})+\mathbf{b} \times \ln (\mathbf{L F}) \text {. }
$$

b renseigne sur le type de croissance de l'espèce considérée :

- $\mathrm{b}=3$, la croissance est isométrique (la croissance en poids est égale à la croissance en taille),

- $\mathrm{b}<3$, le poisson présente une croissance allométrique négative (la croissance en poids est inférieure à la croissance en taille),

- $b>3$, le poisson présente une croissance allométrique positive (la croissance en poids est supérieure à la croissance en taille).

Les histogrammes de la distribution des fréquences de taille ont été établis par site. Les gammes de taille, les classes modales et les tailles moyennes des différentes populations calculées.

\section{Analyses statistiques}

- Le test de $\mathrm{khi}^{2}$ a été utilisé pour comparer les valeurs du sexe ratio observées à la valeur théorique du sexe ratio (1:1).

- Le test $\mathrm{t}$ de Student a été utilisé pour comparer les coefficients allométriques calculés au coefficient allométrique seuil 3.

- $\quad$ Le test ANOVA et le test $\mathrm{t}$ ont été appliqués pour comparer les Kc et les tailles moyennes des catégories d'individus analysées.

Ces tests ont été réalisés à partir du logiciel STATISTICA 7.1.

\section{Résultats}

\section{Evaluation de la pêche}

Effort de pêche

L'effort de pêche varie en fonction du site (Figure 2). Il y a plus de pêcheurs débarquant par jour à Taabo cité et à Courandjourou. Les deux débarcadères ont enregistré en moyenne 14 pêcheurs / j. A Ahondo et à Taabo village, de faibles moyennes d'effort de pêche ont été observées : 6 pêcheurs/ $\mathrm{j}$ à Taabo village et 8 pêcheurs/ $\mathrm{j}$ à Ahondo. L'évolution mensuelle de l'effort de pêche a montré qu'à Taabo cité, beaucoup de pêcheurs ont débarqués au 
cours des mois de décembre (23 pêcheurs/ j), juin (15 pêcheurs/ j) et septembre (19 pêcheurs/ j). En février et mars peu de pêcheurs ont été enregistrés (12 et11 pêcheurs/ j). A Courandjourou le maximum de pêcheurs a été obtenu en mai (17 pêcheurs/ j), et le minimum au mois de septembre avec 11 pêcheurs/ j. A Ahondo, un maximum de 13 pêcheurs/ j a été observé en mars, contre un minimum de 4 pêcheurs/ j en mai. Au cours de l'année, les plus faibles valeurs d'effort de pêche ont été constatées à Taabo village avec un maximum de 8 pêcheurs/ j en janvier, mars et juin et un minimum de 3 pêcheurs/ j en octobre.

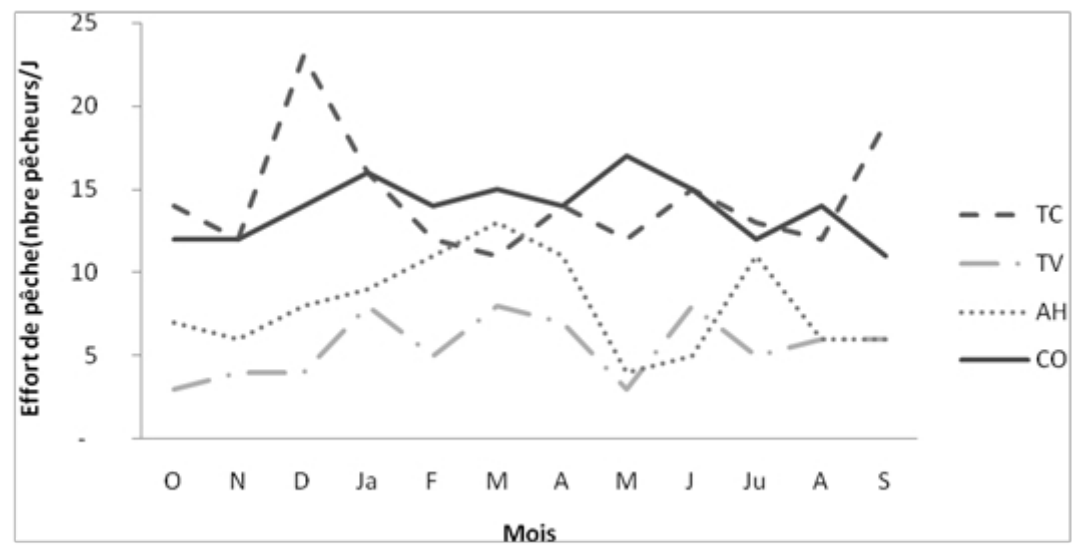

Figure 2. Evolution mensuelle de l'effort de pêche au lac de Taabo d'octobre 2015 à septembre 2016. AH: Ahondo, TC: Taabo cité, TV: Taabo village, CO: Courandjourou

\section{Capture globale}

La Figure 3 montre la répartition des quantités (biomasse) de $S$. punctifer débarquées au lac de Taabo d'octobre 2015 à septembre 2016. La capture globale annuelle de Synodontis punctifer a été estimée à 2199,3 kg. Les quantités sont importantes à Ahondo et à Courandjourou avec près de $1605,3 \mathrm{~kg}$ de poissons débarqués représentant $75 \%$ des captures. Contrairement à ces deux sites, peu de $S$. punctifer sont débarqués à Taabo cité et à Taabo village, seulement $594 \mathrm{~kg}$ débarqués soit $25 \%$ de la capture globale.

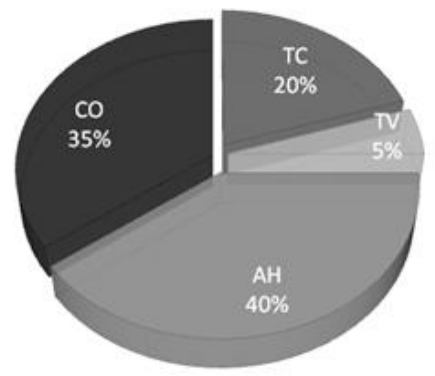

Figure 3. Capture globale de Synodontis punctifer au lac de Taabo d'octobre 2015 à septembre 2016. 
La Figure 4 illustre la variation mensuelle des captures en fonction des zones de pêche. Sur une grande partie de l'année (d'octobre à avril et de juin à septembre) les captures à Ahondo et à Courandjourou sont importantes par rapport à celles de Taabo village et Taabo cité. A Ahondo, la période la plus abondante a été les mois d'octobre et février (plus de $100 \mathrm{~kg}$ ). Les captures ont été faibles en janvier (18 kg) et juin $(15,5 \mathrm{~kg})$. A Courandjourou, si mars a été le mois où les captures ont été importantes $(105 \mathrm{~kg})$, c'est en décembre que les captures de Synodontis ont été peu propices $(15 \mathrm{~kg})$. A Taabo cité, les captures de Synodontis ont été les plus abondantes en mai $(94 \mathrm{~kg})$, et moins débarquées d'octobre à février. A Taabo village, aux mois d'octobre, janvier et avril les captures ont été importantes (plus de $20 \mathrm{~kg}$ ). Les poissons sont très faiblement débarqués le reste de l'année. Par ailleurs, sur ces deux derniers sites les captures mensuelles n'ont pas excédé $100 \mathrm{~kg}$.

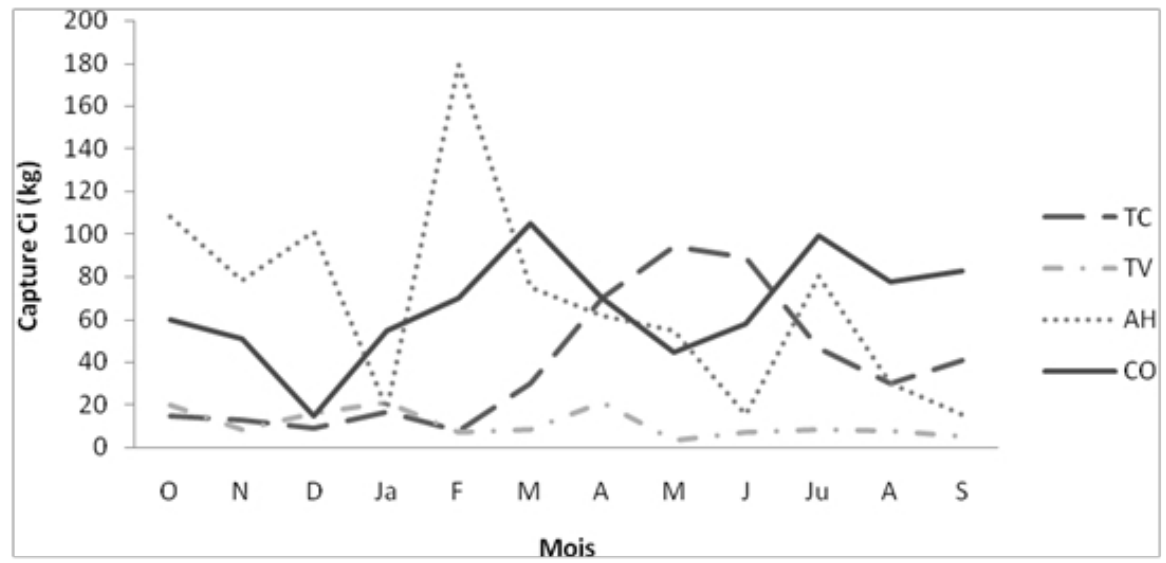

Figure 4. Evolution mensuelle des captures mensuelles (Ci) d'octobre 2015 à septembre 2016 à Taabo.

\section{Prises par unité d'effort}

Les quantités pondérales de $S$. punctifer débarquées par pêcheur à Ahondo ont été plus importantes sur la majeure partie de l'année comparativement aux autres sites, avec des productions avoisinant les $17 \mathrm{~g} /$ pêcheur (Figure 5). Trois pics de production ont été obtenus en octobre, février et mai avec le plus important en février (17,7 g/ pêcheur). Quant aux petites prises, elles ont été observées en janvier (2,5 g/ pêcheur) et juillet $(3,7 \mathrm{~g} /$ pêcheur). Sur le site de Courandjourou, les prises ont atteint près de $9 \mathrm{~g} /$ pêcheur, notamment en mars et en juillet avec respectivement $8,3 \mathrm{~g} /$ pêcheur et $9,2 \mathrm{~g} /$ pêcheur. La petite prise a été au mois de décembre ( $1,2 \mathrm{~g} / \mathrm{pêcheur})$. A Taabo cité et à Taabo village, les productions sont restées relativement les plus faibles durant notre étude. Les productions ont été en dessous de $9 \mathrm{~g} / \mathrm{pêcheur}$. A Taabo cité, la plus grande et la plus petite valeur des prises ont été obtenues respectivement en mai ( $8,3 \mathrm{~g}$ / pêcheur) et février $(0,7 \mathrm{~g} /$ pêcheur). A Taabo 
village, les quantités débarquées ont été importantes en octobre $(8,3 \mathrm{~g} /$ pêcheur) mais faibles en mai, juin et juillet ( 1 g/ pêcheur).

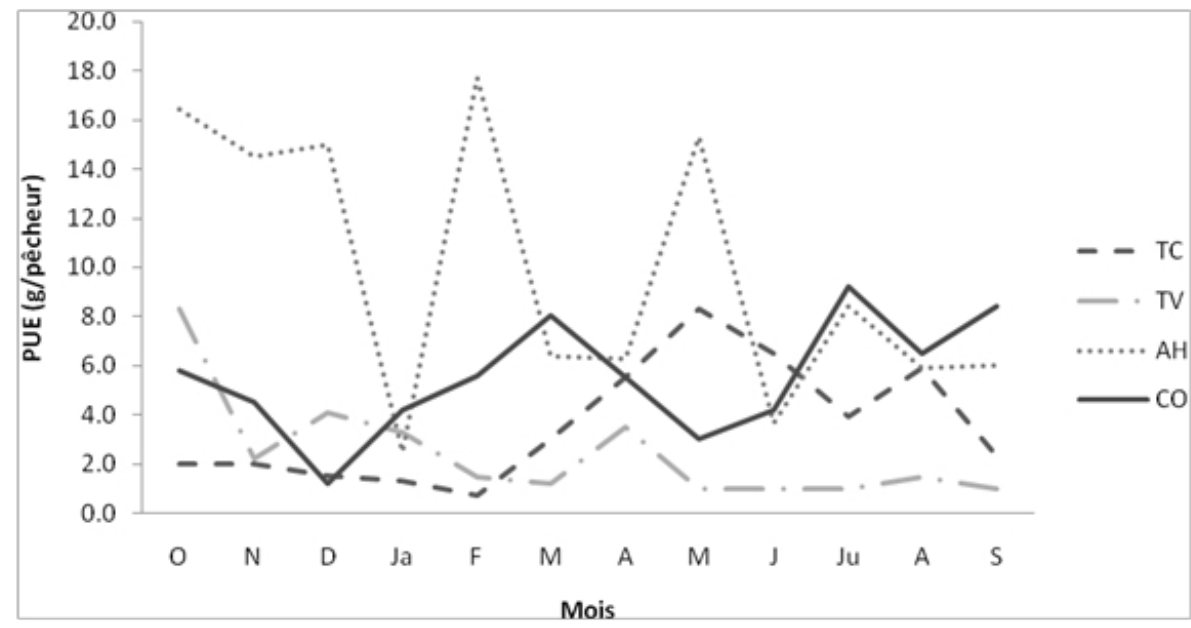

Figure 5. Evolution mensuelle de prises par unité d'effort (PUE) par site à Taabo d'octobre 2015 à septembre 2016.

\section{Caractéristiques morphométriques des populations} Sexe ratio

Sur l'ensemble du lac, un total de 1116 individus a été récolté, reparti en 496 mâles et 664 femelles. Le sexe ratio obtenue est 1:1,33 en faveur des femelles (Tableau I). En fonction des sites, une variation du sexe ratio a été observée. Sur les sites de Taabo cité, de Taabo village et d'Ahondo, le sexe ratio a été en faveur des femelles. Les sexes ratio sont 1:1,15 pour Taabo cité, 1:1,11 pour Ahondo et 1:1,32 pour Courandjourou. Ces valeurs ne diffèrent pas de façon significative de la valeur théorique 1:1. A Taabo village, 91 mâles et 185 femelles ont été identifiés ce qui correspond à un sexe ratio 1: 2,03 en faveur des femelles. Le test de $\mathrm{khi}^{2}$ est significatif à Taabo village.

Tableau I. Sexe ratio dans la population de Synodontis punctifer du lac Taabo d'octobre

2015 à septembre 2016

$\mathrm{n}$ : nombre d'individus, $\mathrm{M}$ : nombre de mâles, $\mathrm{F}$ : nombre de femelles.

\begin{tabular}{cccccc}
\hline Catégorie & $\mathrm{N}$ & $\mathrm{M}$ & $\mathrm{F}$ & Sex-ratio $(\mathrm{M}: \mathrm{F})$ & $\chi^{2}$ \\
\hline Lac & 1160 & 496 & 664 & $1: 1,33$ & 24,33 \\
$\mathrm{TC}$ & 262 & 121 & 141 & $1: 1,16$ & 1,53 \\
$\mathrm{TV}$ & 276 & 91 & 185 & $1: 2,03$ & 32,02 \\
$\mathrm{AH}$ & 375 & 177 & 198 & $1: 1,11$ & 1,18 \\
$\mathrm{CO}$ & 247 & 107 & 142 & $1: 1,32$ & 4,92 \\
\hline
\end{tabular}


Coefficient de condition $(\mathrm{Kc})$

Le coefficient de condition de l'ensemble du lac a varié avec un minimum à 0,43 , un maximum à 3,33 et une moyenne de 1,73 $\pm 3,45$ (Tableau II). En fonction des sites, le coefficient de condition a été supérieur à Taabo cité $(1,71 \pm 0,17)$ et à Taabo village $(2,58 \pm 7,68)$. A Ahondo et à Courandjourou, des valeurs inférieures ont été observées : $1,61 \pm 0,14$ pour Ahondo et 1,6 $\pm 0,22$ pour Courandjourou. Cependant, le coefficient de conditionne diffère pas significativement d'un site à l'autre (ANOVA, F=2,79; $\mathrm{p}>0,05)$. Par rapport

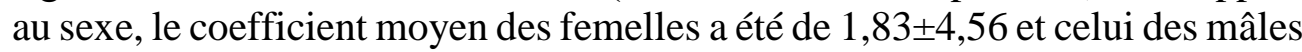
de $1,58 \pm 0,19$. Le test $t$, ne montre pas de différence significative du coefficient de condition entre les deux sexes $(\mathrm{t}=1,58, \mathrm{dl}=1029, \mathrm{p}>0,05)$.

Tableau II. Facteur de condition (Kc) de la population de S. punctifer du lac Taabo d'octobre 2015 à septembre 2016

\begin{tabular}{ccccc}
\hline Catégorie & \multicolumn{3}{c}{$\mathrm{Kc}$} \\
\cline { 2 - 5 } & $\mathrm{n}$ & $\min$ & $\max$ & $\operatorname{moy}$ \\
LAC & 1160 & 0,43 & 3,33 & $1,73 \pm 3,45$ \\
$\mathrm{TC}$ & 262 & 0,69 & 2,22 & $1,71 \pm 0,17$ \\
$\mathrm{TV}$ & 276 & 0,78 & 2,43 & $2,58 \pm 7,68$ \\
$\mathrm{AH}$ & 375 & 1,26 & 2,12 & $1,61 \pm 0,14$ \\
$\mathrm{CO}$ & 247 & 0,43 & 3,3 & $1,6 \pm 0,22$ \\
$\mathrm{~F}$ & 664 & 0,78 & 2,93 & $1,83 \pm 4,56$ \\
$\mathrm{M}$ & 496 & 0,12 & 2,18 & $1,58 \pm 0,19$ \\
\hline
\end{tabular}

n: nombre d'individus, min: minimale, max: maximale, moy: moyenne

\section{Relation allométrique}

Sur l'ensemble du lac, le coefficient d'allométrie a été de 2,89 $\pm 0,03$ (Tableau III). Sur les différents sites de débarquement, les coefficients obtenus

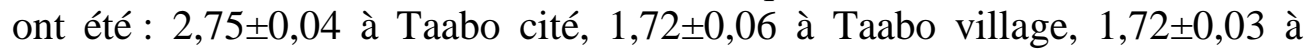
Ahondo et 1,72 $\pm 0,05$ à Courandjourou. Les individus récoltés sur ces différents sites ont une allométrie négative (test $t$ de Student, $\mathrm{p}<0,05$ ). Les mâles ont un coefficient de 1,72 $\pm 0,05$ identique à celui des femelles. Le test $\mathrm{t}$ de Student $(\mathrm{p}<0,05)$ indique que l'allométrie est négative pour ces deux catégories d'individus.

Tableau III : Statistiques descriptives relatives à la taille (LF) et au poids (PT) des spécimens de Synodontis punctifer et paramètres des relations longueur-poids de différentes catégories d'individus d'octobre 2015 à septembre 2016.

\begin{tabular}{|c|c|c|c|c|c|c|c|c|c|c|c|c|}
\hline \multirow{2}{*}{ Catégorie } & \multicolumn{4}{|c|}{$\mathrm{LF}(\mathrm{cm})$} & \multicolumn{4}{|c|}{$\mathrm{PT}(\mathrm{g})$} & \multicolumn{5}{|c|}{ Paramètre de croissance } \\
\cline { 2 - 15 } & $\mathrm{n}$ & $\min$ & $\max$ & $\operatorname{moy}$ & $\min$ & $\max$ & $\operatorname{moy}$ & $\mathrm{B}$ & Es(b) & $\mathrm{a}^{*}(\mathrm{E}-05)$ & $\mathrm{r}^{2}$ & Croissance \\
\hline LAC & 1166 & 8 & 21 & 13,58 & 9,68 & 143,28 & 42,94 & $2,89 \pm 0,03$ & $2^{*} 10^{-3}$ & 0,27 & 0,94 & $\mathrm{~A}^{-}$ \\
\hline TC & 262 & 9,5 & 16,5 & 12,37 & 14,61 & 78,72 & 33,23 & $2,75 \pm 0,04$ & $4 * 10^{-3}$ & 0,22 & 0,86 & $\mathrm{~A}^{-}$ \\
\hline TV & 276 & 8 & 19 & 12,58 & 9,86 & 112,25 & 32,69 & $1,72 \pm 0,06$ & $4 * 10^{-3}$ & 0,66 & 0,91 & $\mathrm{~A}^{-}$ \\
\hline AH & 375 & 10,5 & 21 & 15,38 & 15,1 & 143,28 & 60,19 & $1,72 \pm 0,03$ & $1 * 10^{-3}$ & 0,19 & 0,92 & $\mathrm{~A}^{-}$ \\
\hline CO & 247 & 10 & 19 & 13,63 & 15,92 & 100,12 & 41,97 & $1,72 \pm 0,05$ & $3 * 10^{-3}$ & 0,24 & 0,85 & $\mathrm{~A}^{-}$ \\
\hline F & 664 & 8 & 21 & 13,70 & 9,68 & 143,28 & 44,67 & $1,72 \pm 0,04$ & $1^{*} 10^{-3}$ & 0,32 & 0,94 & $\mathrm{~A}^{-}$ \\
\hline
\end{tabular}




\begin{tabular}{|l|c|c|c|c|c|c|c|c|c|c|c|c|}
$\mathrm{M}$ & 496 & 8,5 & 19 & 13,44 & 9,84 & 112,85 & 40,58 & $1,72 \pm 0,05$ & $2^{*} 10^{-3}$ & 0,26 & 0,89 & $\mathrm{~A}^{-}$ \\
\hline
\end{tabular}

n: nombre d'individus, a: constante de proportionnalité, b: coefficient allométrique, Es: erreur sur $\mathrm{b}, \mathrm{r}^{2}$ : coefficient de détermination, A-: croissance allométrique négative

\section{Structures de taille}

La structuration de la population de $S$. punctifer (Tableau IV) a montré que les poissons débarqués à Ahondo ont une large gamme de taille de [LF = 10-22 cm]. A Courandjourou, une large gamme de taille a également été observée mais à un degré moindre par rapport à Ahondo [LF $=10-21 \mathrm{~cm}]$. A Taabo cité et à Taabo village, les gammes de taille des poissons sont plus réduites par rapport aux deux précédentes zones. La taille des poissons a oscillé entre 8 et $16 \mathrm{~cm}$ à Taabo village et entre 9 et $17 \mathrm{~cm}$ à Taabo cité.

La taille moyenne des individus d'Ahondo a été plus grande avec une moyenne $=15,38 \pm 1,68 \mathrm{~cm}$. Dans la zone de Courandjourou, les individus ont une taille intermédiaire $(\operatorname{moy}=13,63 \pm 1,64 \mathrm{~cm})$. A Taabo cité et à Taabo village, les tailles moyennes observées ont été les plus petites : 12,37 $\pm 1,24 \mathrm{~cm}$ pour Taabo village et $12,58 \pm 1,58 \mathrm{~cm}$ pour Taabo cité. Le test $\mathrm{t}(\mathrm{t}=3,23, \mathrm{dl}=$ 2095, $\mathrm{p}<0,05)$ n'a pas montré de différence significative entre les tailles moyennes des individus de Taabo cité et Taabo village. Les tailles moyennes des individus d'Ahondo, de Courandjourou et des deux sites de Taabo pris ensemble sont significativement différentes (ANOVA, F=8,64; p > 0,05).

A Ahondo, la classe modale obtenue [15-18] a été supérieure à celle de Courandjourou, Taabo cité et Taabo village où la classe modale est restée identique [12-13].

Tableau IV. Structure de taille des populations de S. punctifer au lac de Taabo d'octobre 2015 à septembre 2016.

\begin{tabular}{|c|c|c|c|}
\hline Catégorie & Gamme de taille & Taille moyenne LF $(\mathrm{cm})$ & Classe modale \\
\hline $\mathrm{AH}$ & {$[10-22]$} & $15,38 \pm 1,68$ & {$[15-18]$} \\
\hline $\mathrm{Co}$ & {$[10-21]$} & $13,63 \pm 1,64 \mathrm{~cm}$ & {$[12-13]$} \\
\hline $\mathrm{TV}$ & {$[8-16]$} & $12,37 \pm 1,24 \mathrm{~cm}$ & {$[12-13]$} \\
\hline $\mathrm{TC}$ & {$[9-17]$} & $12,58 \pm 1,58 \mathrm{~cm}$ & {$[12-13]$} \\
\hline
\end{tabular}




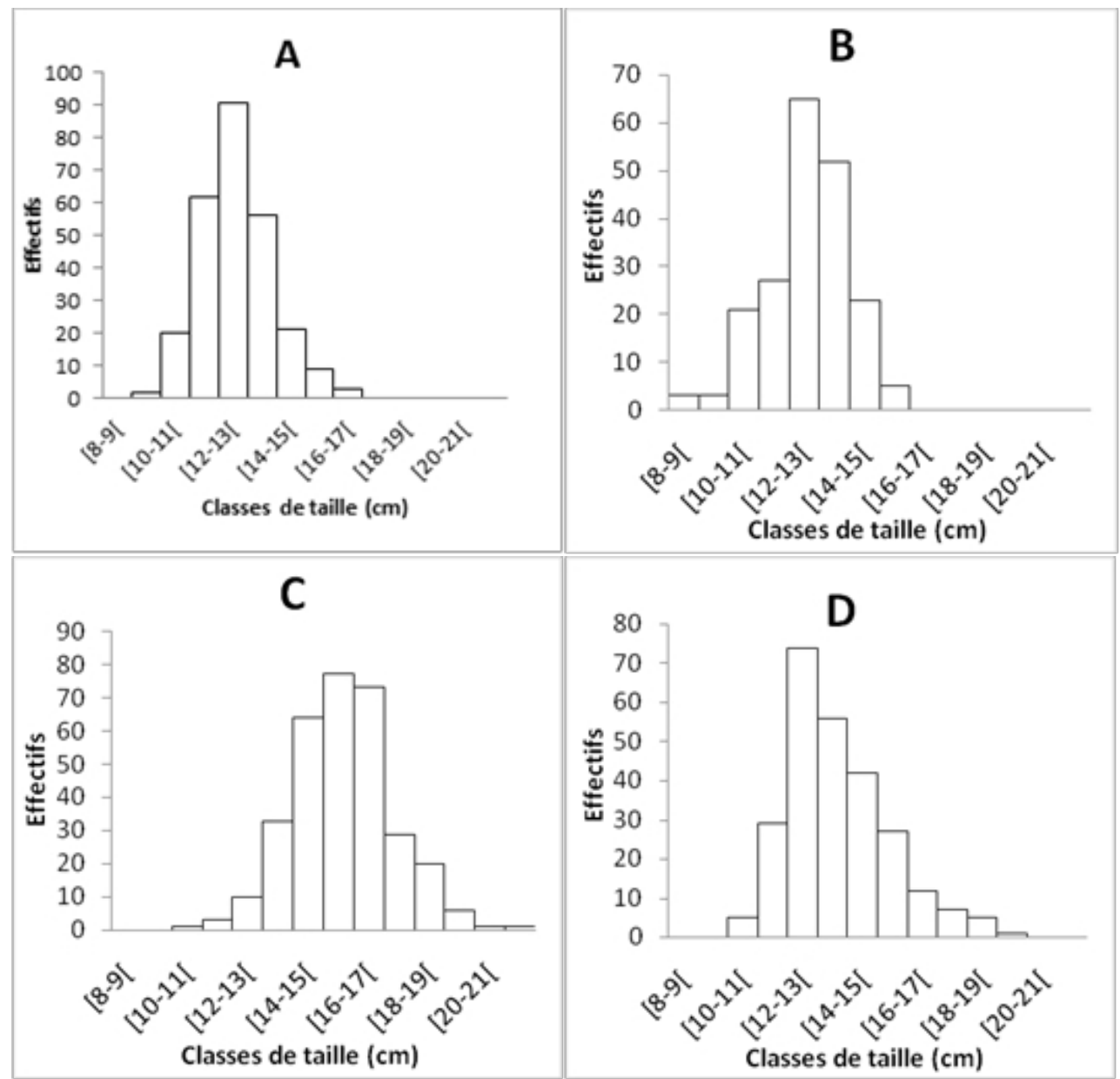

Figure 6. Histogrammes des fréquences de taille de Synodontis punctifer du lac de Taabo d'octobre 2015 à septembre 2016. A : Taabo cité, B : Taabo village, C : Ahondo, D : Courandjourou

\section{Discussion}

L'étude de la pêche et des caractéristiques morphologiques de Synodontis punctifer a mis en évidence deux zones de pêche dans le lac de Taabo. Une zone à fort effort de pêche : Taabo cité et Courandjourou et une zone à faible effort de pêche: Ahondo et Taabo village. Cela pourrait s'expliquer par la catégorisation des pêcheurs sur les zones de pêche. D'un côté, il y a les pêcheurs étrangers à Taabo cité et à Courandjourou. Ces pêcheurs «bozos» ont pour seule activité la pêche. Ce qui fait qu'on les retrouve quasiment toute l'année sur le lac en très grand nombre. Ces derniers dominent l'activité de pêche sur deux barrages (Ayamé et Buyo) en Côte d'ivoire (Vanga, 2014). A Taabo, 90,99\% des pêcheurs professionnels sont allogènes (Koudou, 2012). De l'autre, il y a les pêcheurs autochtones dans les villages d'Ahondo et de Taabo village. Ces pêcheurs, en général planteurs, 
pratiquent la pêche occasionnellement, d'où leur faible nombre sur le lac. Les pêcheurs professionnels ivoiriens représentent $9,01 \%$ des pêcheurs au lac de Taabo (Koudou, 2012).

De plus à Taabo village, le manque de pêcheurs se justifierait aussi du fait qu'un bon nombre de pêcheurs s'intéressent à la pêche des Pellonula leonensis avec non pas les filets maillants mais les sennes de rivage comme engin de captures. Cette remarque peut se confirmer grâce aux études de Kraidy et al. (2014). Ces auteurs ont enregistré, avec les sennes de plage, 3547 sorties pêche/an pour une production de 211,860 tonnes de Pellonula leonensis entre 2008 et 2009.

Les captures globales montrent une dominance des quantités débarquées à Ahondo et à Courandjourou, contre un faible niveau de production à Taabo cité et Taabo village. Cela confirme les résultats des PUE obtenus. Les végétaux dans la zone d'Ahondo et de Courandjourou pourraient en être la cause. Lauzanne (1988) constate un régime alimentaire riche en débris végétaux, débris animaux chez les Synodontis. Les $S$. punctifer seraient donc attirés vers Ahondo, et Courandjourou où la présence des végétaux est une importante source de nourriture. Cette remarque est soutenue par Lévêque (1995) qui indique que la recherche de nourriture est un élément déterminant dans le choix de l'habitat.

Les valeurs calculées du coefficient d'allométrie b par rapport aux différents sites d'étude du lac Taabo ainsi qu'au sexe sont inférieures à 3, ce qui suggère que $S$. punctifer a une croissance allométrie négative sur l'ensemble du lac. Des résultats similaires ont été observés chez Synodontis schall et Synodontis nigrita au Bénin (Lalèyè et al., 2006). Les Synodontis semblent croitre plus en taille qu'en poids.

Sur les sites d'Ahondo, de Courandjourou et de Taabo village, le nombre de mâles était le même que celui des femelles, contrairement à Taabo village où deux fois plus de femelles que de mâles ont été identifiées. Les mâles pourraient être attirés par les femelles mâtures présentent à Ahondo et Courandjourou. Cette différence de migration entre mâles et femelles est corroborée par les travaux de Kouassi et al. (2013).

Le facteur de condition $(\mathrm{Kc})$ observé selon le site d'échantillonnage et du sexe ne varie pas de manière significative. L'espèce semble s'adapter aux différents milieux de vie. Son régime alimentaire très varié serait un avantage pour sa survie dans ces différents milieux. Ces résultats sont similaires à ceux de Diomandé et al. (2001), qui constate un régime alimentaire de type omnivore chez Synodontis schall dans la Bia.

La répartition des populations pourrait montrer une zone de frayage de l'espèce sur le à Taabo. En effet les individus de grandes tailles observés à Ahondo semblent indiquer que cette zone est la zone de reproduction de $S$. punctifer sur le lac. Cette zone présente l'avantage d'être une excellente zone 
d'alimentation. De plus, la présence d'herbe empêche les activités de pêche. Ce même constat a été fait sur le lac Taabo par (Aliko et al., 2010). Ils ont montré que la partie supérieure du lac présente une importante zone d'inondation qui constitue des frayères potentielles pour $D$. rostratus.

Les structures de taille ont mis en évidence trois zones de pêche caractérisées par trois groupes d'individus: la zone Ahondo avec des individus de grande taille, la zone Courandjourou avec des individus de taille intermédiaire et la zone Taabo cité - Taabo village avec des individus de petite taille. Cela expliquerait un phénomène de migration de S. punctifer à Taabo. En effet les juvéniles rencontrés à Taabo cité et à Taabo village en aval migrent vers la zone de reproduction à Ahondo constituant la partie fluviale du lac. Courandjourou, zone de rencontre entre le fleuve et le lac, et où l'on rencontre des juvéniles avant la maturation, serait la zone de transit. Ces observations sont en accord avec celles obtenues sur $S$. schall qui, dans les grands lacs africains, migrent dans des petits affluents temporaires pour se reproduire (Hopson, 1982).

\section{Conclusion}

Cette étude a permis de montrer que $S$. punctifer est une espèce peu débarquée au lac de Taabo. La production globale est de 2199,3 kg/ an. Les sites d'Ahondo et de Courandjourou sont les zones où ils abondent. Le sexe ratio $(1: 1,33)$ est en faveur des femelles. Le coefficient allométrique b est inférieur à $3, S$. punctifer croit donc plus en taille qu'en poids. Le facteur de condition, qui ne diffère pas significativement sur l'ensemble du lac, indique l'adaptation de S. punctifer aux différents milieux écologiques du lac. Il existe une migration de l'espèce dans le lac : les individus de petite taille capturés à Taabo cité et Taabo village migrent vers la zone de reproduction d'Ahondo en transitant par Courandjourou.

\section{References:}

1. Aliko, N. G., Da Costa, K. S., Konan, K. F., Ouattara, A., \& Gourène, G. (2010). Fish diversity along the longitudinal gradient in a lake of West Africa, Taabo hydroelectric reservoir, Ivory Coast. Ribarstvo, 68 (2) : 47-60.

2. Berté, S., Kouamélan, E.P., Ouattara, N.I., Koné T., Goore B.G., N'Douba V., \& Kouassi, N. J. (2008). Cycle de reproduction et fécondité de Distichodus rostratus (Characiformes, Distichodontidae) dans un bassin ouest africain (fleuve Bandama, Côte d'Ivoire). Tropicultura, 26 (2) : 104-107.

3. Da Costa, K. S. \& Dietoa, Y. M. (2000). Typologie de la pêche sur le lac Faé (Côte d'Ivoire) et implications pour une gestion rationnelle des ressources halieutiques. Bull. Fr. Pêche Piscic., 384 : 1 - 14. 
4. Diaha, N. C., N'Da, K., \& Kouassi, K.D. (2000). Etude comparée de la pêche des thonidés mineurs par les chaluts doubles et les pirogues dans la zone économique exclusive (ZEE) ivoirienne. Tropicultura, 27 (3) :152-158.

5. Diomandé, D., Gourène, G., \& Luis Tito de Morais, (2001). Stratégies alimentaires de Synodontis bastiani (siluriformes : Mochokidae) dans le complexe fluvio-lacustre de la Bia, Côte d'Ivoire. Cybium, 25 (1) : 7-21

6. Garcia, S. \& Demetropoulos, A. (1986). L'aménagement de la pêche à Chypre. FAO Document Technique Pêches, $250: 43$ p.

7. Gerlotto, F. \& Stequert, B. (1978). La pêche maritime artisanale en Afrique de l'Ouest. Caractéristiques générales. La Pêche Maritime, $1202: 278-285$

8. Groga, N. (2012). Structure, fonctionnement et dynamique du phytoplancton dans le lac de Taabo (Côte d'Ivoire). Thèse de doctorat de l'université de Toulouse, $224 \mathrm{P}$.

9. Hopson, A. J. (1982). Lake Turkana. A report on the findings of the Lake Turkana. Project 1972-1975. London Overseas Development Administration, $6: 1614 \mathrm{p}$.

10. Kartas, F. \& Quignard, J.-P. (1984). La fécondité des poissons téléostéens. Masson (Collection biologie des milieux marins), Paris, $117 \mathrm{p}$.

11. Kouassi, K. L., Gone, D. L., Mélédjé, N. H., Wognin, A. V., \& Aka, K. (2007). Hydrologie et évolution spatio-temporelle des charges solides en suspension dans le lac du barrage hydroélectrique de Taabo (Côte d'Ivoire). EJSR, 18 (3) : 464 - 478.

12. Kouassi, S. K., Koné, A., Akadjé, C. M. A., Diaby M., \& N’Da, K. (2013). Ecologie des poissons capitaines: Polydactylus quadrifilis (Cuvier, 1829), Galeoides decadactylus (Bloch, 1795) et Pentanemus quinquarius (Linné, 1758) de la pêcherie artisanale maritime de Grand-Lahou (Côte d'Ivoire).Tropicultura, 31 (3) :187-193.

13. Koudou, D. (2012). Pêche et développement socioéconomique : cas de la sous-préfecture de Taabo (Côte d'Ivoire). Thèse unique de doctorat, Université Félix Houphouët-Boigny de Cocody (Abidjan, Côte d'Ivoire), $349 \mathrm{p}$.

14. Koudou, D. (2014). Eaux et conflits en Côte d'Ivoire : regard géographique sur des conflits halieutiques larvés au lac du barrage hydroélectrique de Taabo. Revue Canadienne de Géographie Tropicale, 2 (1) : 47-56.

15. Kraidy, L. A. B., Koné, N., Berté, S., N'zi, G.K, Yao, S. S., \& Kouamelan, P. E. (2014). Pêche et paramètres de reproduction de Pellonula leonensis Boulenger 1916, dans le lac de Taabo (Fleuve 
Bandama, Côte d'Ivoire) : implications pour une exploitation durable du stock. Int. J. Biol. Chem. Sci., 8 (1): 75-88.

16. Laë, R. (1997). Estimation des rendements de pêche des lacs africains au moyen de modèles empiriques. Aquat. Living. Resour., 10 : 83 -92.

17. Lalèyè, P., Chikou, A., Gnohossou, P., Vandewalle, P., Philippart, J. C., \& Teugels, G. (2006). Studies on the biology of two species of catfish Synodontis schall and Synodontis nigrita (Ostariophysi : Mochokidae) from the Ouémé River Bénin. Belg. J. Zool., 136 (2) : 193-201.

18. Lauzanne, L. (1988). Les habitudes alimentaires des poissons d'eau douce africains. In : Orstom (Ed.).Biologie et écologie des Poissons d'eau douce africains (pp. 395-425). Paris.

19. Lévêque, C. (1995). L'habitat : être au bon endroit au bon moment? Bull.fr. Pêche Pisc. 9 (20) :337-339.

20. Lévêque, C. \& Paugy, D. (1999). Impacts des activités humaines. In : IRD (Ed.), Les poissons des eaux continentales africaines : diversité, biologie, écologie, utilisation par l'homme (pp : 365-383).Paris.

21. LE Cren, E.D. (1951). The length-weight relationship and seasonal cycle in gonad weight and condition in the perch (Perca fluviatilis). J. Anim. Ecol., 20: 201-219.

22. Micha, J.C. \& Franck, V. (2004). Etude prospective pour la relance $d u$ secteur pêche et aquaculture en côte d'Ivoire. Ministère de la Production Animale et des Ressources Halieutiques (60 p). Abidjan.

23. Paugy, D., \& Roberts, T. R. (1994). Mochokidae. In Orstom (Ed.), Faunes des poisons d'eau douce et saumâtres de l'Afrique de l'Ouest (pp : 500-563). Paris.

24. Poll, M. (1971). Révision des Synodontis Africains (Famille Mochokidae). In : A. Pinton, 2006. Anatomie, systématique et phylogénie de poissons Synodontis actuels et fossiles (Siluriformes, Mochokidae) : implications dans la paléobiogéographie intracontinentale néogène d'Afrique. Thèse de doctorat de l'université de Poitier, $280 \mathrm{p}$.

25. Tah, L., Da Costa, K. S., Kouassi, J. N., \& Moreau, J. (2009). Effort de pêche et production piscicole au lac d'Ayamé I (bassin de la Bia, Côte d'Ivoire) après le départ des pêcheurs $<<$ bozos $>>$. Agronomie Africaine 21 (1) : 103-115.

26. Vanga, AF. (2004). Conséquences socio-économiques de l'expulsion des pêcheurs étrangers en Côte d'ivoire : lacs d'Ayamé et de Buyo. Revue européenne des migrations internationales, 20 (1) : 197-205.

27. Vanga, A.F. (2011). Evolution de la pêche au lac d'Ayamé depuis l'expulsion des pêcheurs non nationaux (Côte d'Ivoire). Tropicultura, 29 (1) : 8-13. 